\title{
The Formation and Evolution of the Sun and the Source of Star Energy as Well as the Sunspots and Flares of the Sun
}

\author{
Cuixiang Zhong \\ Jiangxi Normal University, Nanchang 330022, China
}

\begin{abstract}
Nebula theory is the most widely accepted hypothesis about the formation and evolution of the Solar System. This theory holds that the Sun is formed from a collapsed gas cloud 4.57 billion years ago; when the core temperature of the gas cloud rises to 10 million $\mathrm{K}$, the thermonuclear reaction of hydrogen fusion into helium is ignited, then the Sun become a star; once the hydrogen in the core is exhausted, the life of the star will end. But the limited hydrogen element obviously cannot satisfy such a long-term thermonuclear reaction, in order to sustain long-term thermonuclear reactions, a steady stream of fuel must be obtained from space. So the existing hypothesis about the formation and evolution of the Solar System has serious defects. Thus the author has studied the formation of the Moon, the Earth and the Sun, and discovered the formation of the Sun and the real source of star energy. The author could also explain many solar activity phenomena such as sunspots, flares, prominences, etc.
\end{abstract}

Key words: Sun, formation, evolution, star energy, sunspots, flares.

\section{Introduction}

Various hypotheses have been proposed about the formation and evolution of the Sun [1, 2]. Among these hypotheses, the widely accepted hypothesis is the Nebular Hypothesis, which holds that the Sun formed from a collapsed nebula 4.57 billion years ago; a rotating nebula underwent gravitational collapse, when the core temperature of the nebula rose to 10 million $\mathrm{K}$, the thermonuclear reaction of hydrogen fusion into helium was ignited, releasing a great deal of energy, causing the ambient temperature and pressure to rise, thus the surrounding hydrogen also began to fuse, from near to far, a series of thermonuclear reactions were carried out, finally the Sun became a star that emits light and heat; once the hydrogen in the core is exhausted, the life of the star will end. But according to scientific estimates, the

Corresponding author: Cuixiang Zhong, Dr., Associate Professor, research fields: astrophysics and computer application. thermonuclear reaction of the Sun has lasted for about 4.57 billion years, and it will last more than 5 billion years, the limited hydrogen element obviously could not satisfy such a long-term thermonuclear reaction, in order to sustain long-term thermonuclear reactions, a steady stream of fuel must be obtained from space. So the existing hypothesis of solar formation has serious defects. Thus the author has studied the formation and evolution of the Moon, the Earth and the Sun, and discovered the formation and evolution of the Sun and the real source of star energy. The author could also explain many solar activity phenomena such as sunspots, flares, prominences, etc.

\section{The Formation and Evolution of the Sun}

Since the Sun revolves around the centre of the Milky Way Galaxy, it must have been a satellite produced by a parent star, just like the Moon produced by the Earth. Therefore, the evolution of the Sun from the original satellite to the main-sequence star of today has gone through many stages. 


\subsection{From a Satellite to a Planet}

When the early Sun was a moon-sized satellite, its orbit was very close to its parent planet, so it was almost synchronously tidally locked with the parent planet, unable to rotate on its own axis. But with the frequent collisions of prograde planetesimals or the accelerating rotation of its parent planet due to the constant contraction of the parent planet, its revolution speed increased accordingly, therefore it moved outward, expanding the distance between it and its parent planet. In addition, during the satellite's revolution around its parent planet, it constantly accreted nebular material near its orbit to become larger and larger. With the continuous increase in the mass and volume of the satellite, the heat inside the satellite accumulated continuously. It is just the original energy accumulated to a certain extent that made the inner material melted and differentiated, forming the different layers of the satellite: crust, mantle and nucleus [3].

When the satellite grew up into a planet like the Earth, it was large enough to absorb much vapor and other gases from the cosmic space to form an extended atmosphere and even a large hydrosphere. When its parent star became a star constantly giving off light and heat, since the planet moved counter-clockwise around its parent star, the planet's hemisphere near the parent star was exposed to the "sunlight" from the parent star, so the temperature of this hemisphere was generally higher than that of the other, making more vapor evaporated from this hemisphere than from the other, even forming massive clouds impacting high mountains or massive storms blocking the planet's revolution in this hemisphere. Hence, during the planet's revolution around its parent star, the planet's hemisphere near the parent star encountered more air resistance than the other hemisphere, causing the planet to rotate from west to east $[4,5]$.

Due to the effects of the centrifugal force of the planet's rotation, the planet grows into an equatorial bulge and a slightly flat sphere at both poles. The equatorial radius of this planet is larger than polar radii and other radii, and the gravitational force is inversely proportional to the square of the distance, so the nearer the object is to equatorial region, the smaller the gravitational force applied on the object is. Hence, the erupted objects from equatorial region can easily achieve high enough speed to enter some orbits around the planet. In addition, the equatorial region receives more radiation from the parent star and evaporates more water vapor than other places, therefore has more rain, while high latitudes have lower temperatures and less rain. Hence, the equatorial region suffered water erosion much earlier and more serious than other places, therefore this region had the earliest and most violent volcanic eruptions of the planet. During some violent volcanic eruptions, some volcanic ash and debris could achieve high enough speed to enter some orbits around the planet, becoming material to condense satellites. In addition, fast-rotating planets have their own polar vortices, which can span troposphere and stratosphere. Dust and volcanic ash from the planet's surface can enter the stratosphere through the jet of polar vortices, continuously supplying materials for satellites formed in the stratosphere. Since the stratosphere is thicker in low latitudes and thinner in high latitudes, satellites orbiting the planet are mostly formed near the equator. That is why the orbits of the eight planets lie in the same plane, and the angle between this plane and the solar equatorial plane is very small $[6,7]$.

\subsection{From a Planet to a Star}

After the original Sun evolved from a satellite of small size and mass into an earth-sized planet, it generated some satellites, but it still revolved around its parent star, unceasingly accreted the nebula materials near the orbits to become larger and larger, and gradually moved away from its parent star with the frequent collisions of prograde planetesimals or the accelerating rotation of its parent star due to 
contraction. Afterwards it met a series of impacts from some other planets running into it from behind, making it become a Jupiter-sized planet much farther away from its parent. Since Jupiter's mass is very huge, it can attract various gas molecules to form a dense atmosphere, and produce strong polar vortices during its rotation. Moreover, this kind of polar vortex can generate strong spiral currents, therefore form strong dipole magnetic fields, as is shown in Fig. 1, which is captured by NASA's Hubble Space Telescope [8].

Although Jupiter's mass is large enough to attract hydrogen in the atmosphere, making the mass ratio of hydrogen in Jupiter reach $75 \%$, which is comparable to the mass ratio of hydrogen in the Sun, yet Jupiter lacks oxidants, therefore cannot burn directly. Jupiter has an internal pressure of about 40 million standard atmospheric pressure, and its inner temperature is $280,000^{\circ} \mathrm{C}$, but it can't be detonated directly into a star. In 1994, humans witnessed a spectacular ends when comet Shoemaker-Levy 9 impacted Jupiter. The collision produced scars that were visible from Earth, but Jupiter hasn't been detonated into a star [9]. According to scientists' estimate, only one giant has a mass 70 to 80 times that of Jupiter can it have enough gravity, pressure and temperature to cause fusion reaction between hydrogen elements. In fact, the proto-sun became a detonable star just by accreting interstellar material near its orbit.

In the past people thought that the Sun formed from a collapsed nebula 4.57 billion years ago. This nebula collapsed due to its own gravitational action, and the temperature and pressure inside the nebula increased continuously during the collapse process. When the core temperature of the nebula rose to 10 million $\mathrm{K}$, the thermonuclear reaction of hydrogen fusion into helium was ignited, releasing a great deal of energy, causing the ambient temperature and pressure to rise, thus the surrounding hydrogen also began to fuse, from near to far, a series of thermonuclear reactions were carried out, finally the Sun became a star releasing immense energy; once the hydrogen in the core is exhausted, the life of the star will end. But according to scientific estimates, the thermonuclear reaction of the Sun has lasted for about 4.57 billion years, and it will last more than 5 billion years, the limited hydrogen element obviously cannot satisfy such a long-term thermonuclear reaction, in order to sustain long-term thermonuclear reactions, a steady stream of fuel must be obtained from space. So the existing hypothesis of solar formation and evolution has serious defects. In fact, before the proto-sun evolved into a star, it was a massive planet, which has a strong gravitational pull and a dense atmosphere.

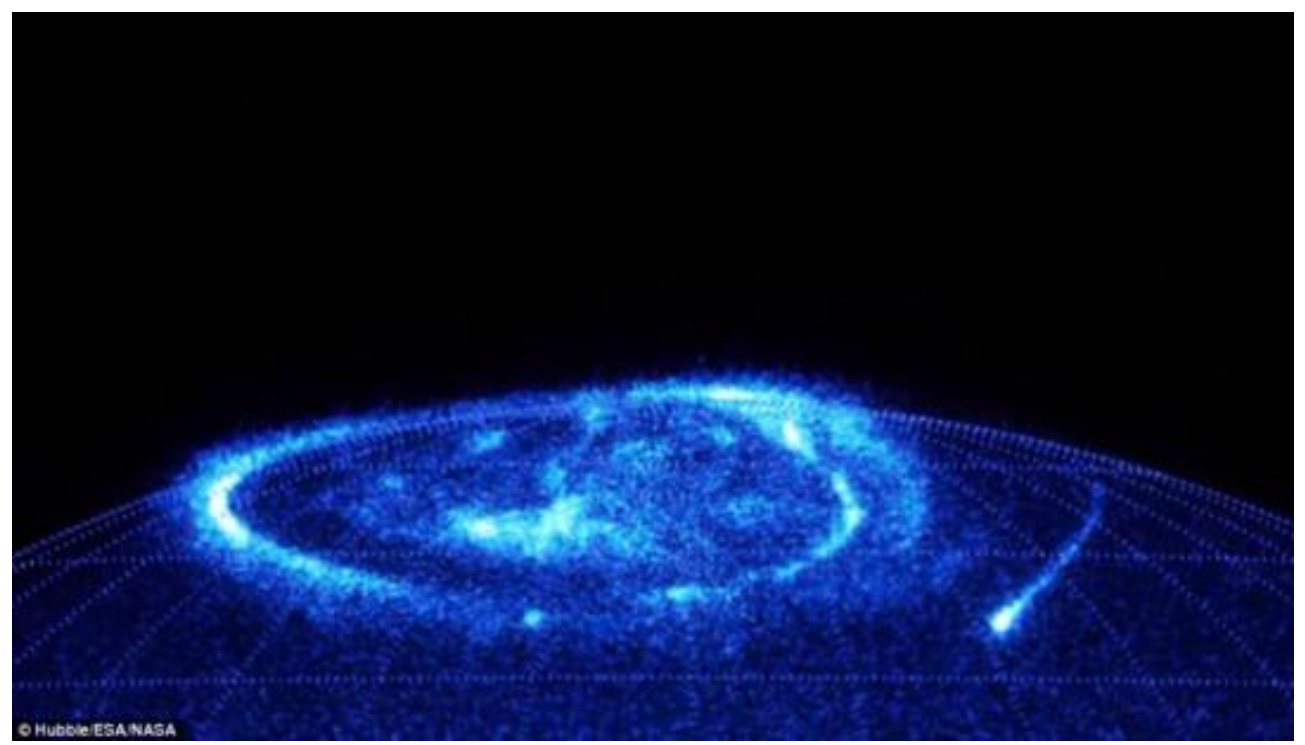

Fig. 1 The spiral current generated by Jupiter's arctic vortex. 
Due to the effect of centrifugal force, the rotation of the proto-sun around its own axis has caused it to bulge around the Equator, making the proto-sun become an oblate spheroid with the radius of the two poles is less than the radius of the equator and other places, while the gravitational force is inversely proportional to the square of the distance. When the proto-sun rotates quickly, the rotation will produce strong centrifugal force, making the clouds over the equator and low latitudes tend to move away from their orbits to the South pole or the North Pole. Because the gravitational attraction of the polar position is greater than that of other locations, when clouds move above the polar regions, they are easily attracted by the gravitational pull of the polar regions, after inhaling cold air, they condense into thick clouds and sink gradually. Many polar-plunging clouds form a strong circulation around the pole as the proto-sun rotates, that is polar vortex, as is shown in Fig. 2. This kind of vortex can continuously absorb hydrogen and other matter from the surrounding space to maintain the thermonuclear reaction on the proto-sun. Originally, the proto-sun has two groups of vortices, located at the South pole and the North pole respectively, which can span troposphere and stratosphere. As clouds swept in by a vortex of the proto-sun sink faster and colder, after a long spiral path, at the bottom of the vortex, the velocity of the airflow is tens of times faster than that of scale 12 typhoon, so the clouds have already condensed into ice, and the temperature in the vortex is much lower than that around it, hence from the distant Earth, the vortex looks like a small sunspot. But in fact, the central depth of the vortex can reach 200,000 kilometers, and its diameter can reach tens of thousands of kilometers.

Since the clouds involved in polar vortex are constantly in flux and revolve downward rapidly in a spiral manner, a series of parallel thick spiral cloud paths can be formed, which facilitate the downward flow of heavier negatively charged cloud droplets, thus forming a series of good circuits with excellent electrical conductivity. Since the clouds involved in polar vortex are numerous and revolve rapidly, it is easy to have violent frictions and collisions among clouds, making the vortexes filled with positive ions and negative ions. Cloud droplets must first absorb negative ions in the atmosphere, causing the droplets to be negatively charged, and the larger cloud droplets fall toward the lower part of the cloud or even the lower portion of the vortex along a spiral cloud path, while the lighter positive ions are gradually brought up by the updraft to the upper part of the cloud or even the upper portion of the vortex along the spiral cloud path, forming a current from the lower portion of the vortex to the upper portion of the vortex along the spiral

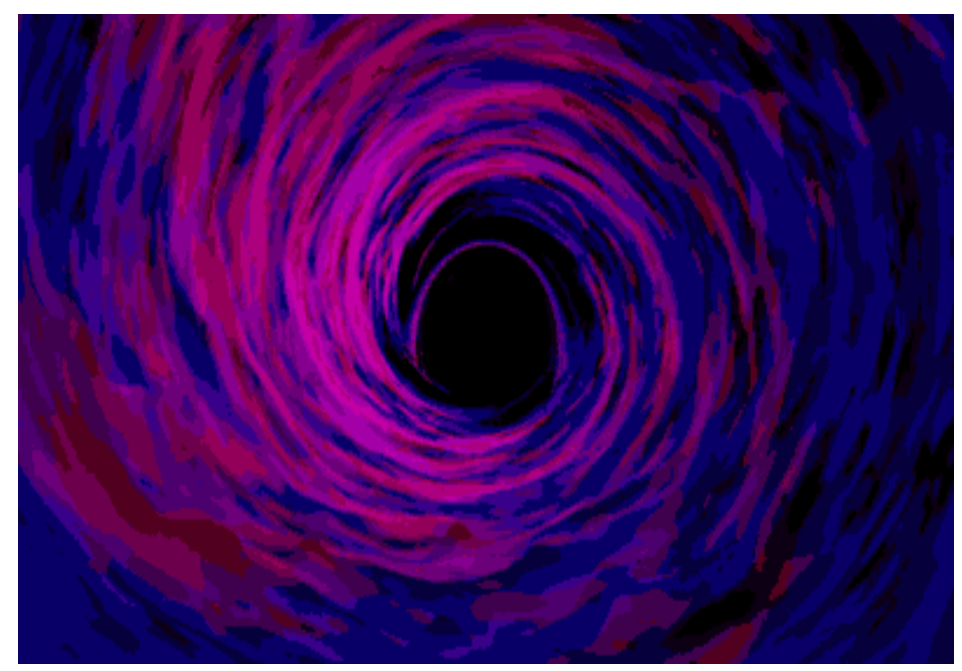

Fig. 2 Polar vortex on the Sun. 
cloud path. In addition, since the clouds along a spiral cloud path are numerous and revolve rapidly, it is easy to have violent frictions and collisions among clouds, producing frequent electrical discharge or thunderstorms. Each electrical discharge or thunderstorm acts as an electrostatic motor, which can send currents to the upper portion or the lower portion of the vortex, preponderantly forming an intense current following the cloud path from the bottom of the vortex to the top of the vortex. Since there is a continuous flow of currents along the spiral cloud path from the bottom of the vortex to the top of the vortex, thus forming a powerful dipole magnetic field with its magnetic north pole pointing towards the south pole of the proto-sun and its magnetic south pole pointing towards the north pole of the proto-sun, as is shown in Fig. 3.

In addition, since the coverage of a polar vortex on the proto-sun is huge, the number of clouds involved by the polar vortex is also very large. In the process of clouds' sinking, there is a long spiral path to go, making clouds bigger and colder, at the bottom of the vortex, the clouds have already condensed into huge crystal bodies. Some crystals are even larger than one earth, so they are prone to severe friction and collision during rapid rotation, leading frequent and violent thunderstorms, thus releasing huge amounts of electricity and heat, even producing violent jets, which is called prominences. For example, famous astronomer Nassim Halamin recently found from an image of SOHO that a white earth-sized object flew out of a sunspot region in the Sun's arctic area, as is shown in Fig. 4.

It is known that a powerful thunderstorm on Earth can generate billions to hundreds of billions of watts of electricity, and make the temperature of the ambient air rise rapidly to $10,000-20,000^{\circ} \mathrm{C}$, while the diameter of the proto-sun is 106 times that of the earth, the volume of the proto-sun is about 1.3 million times that of the Earth, and the central pressure is 300 billion atmospheric pressure, so the volume of an atmospheric vortex on the proto-sun is more than 1 million times that of an atmospheric vortex on the Earth, consequently, a powerful thunderstorm generated

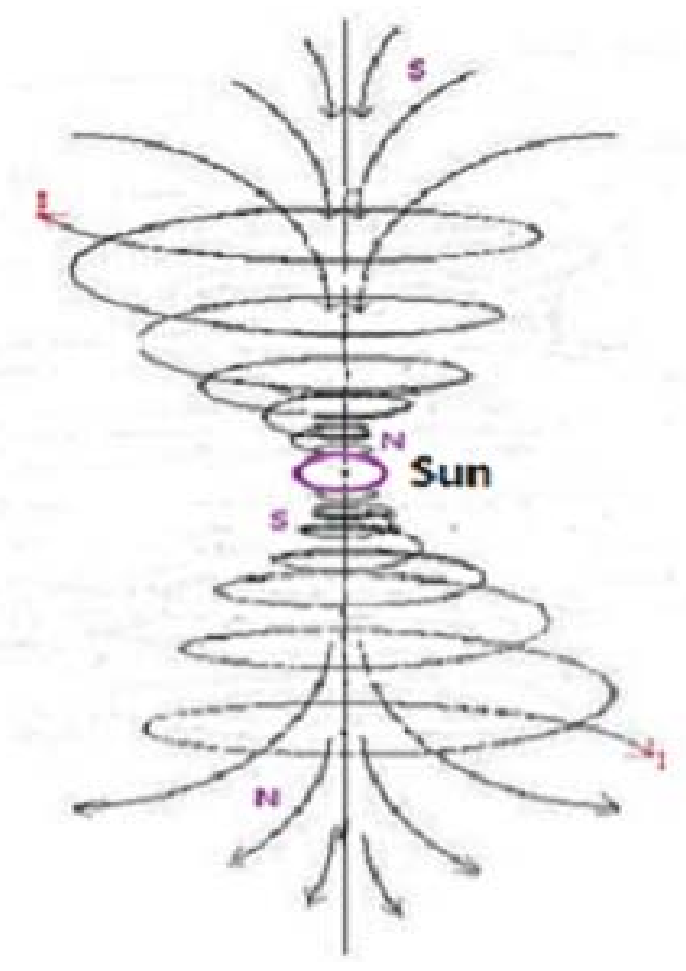

Fig. 3 Magnetic field of vortex. 


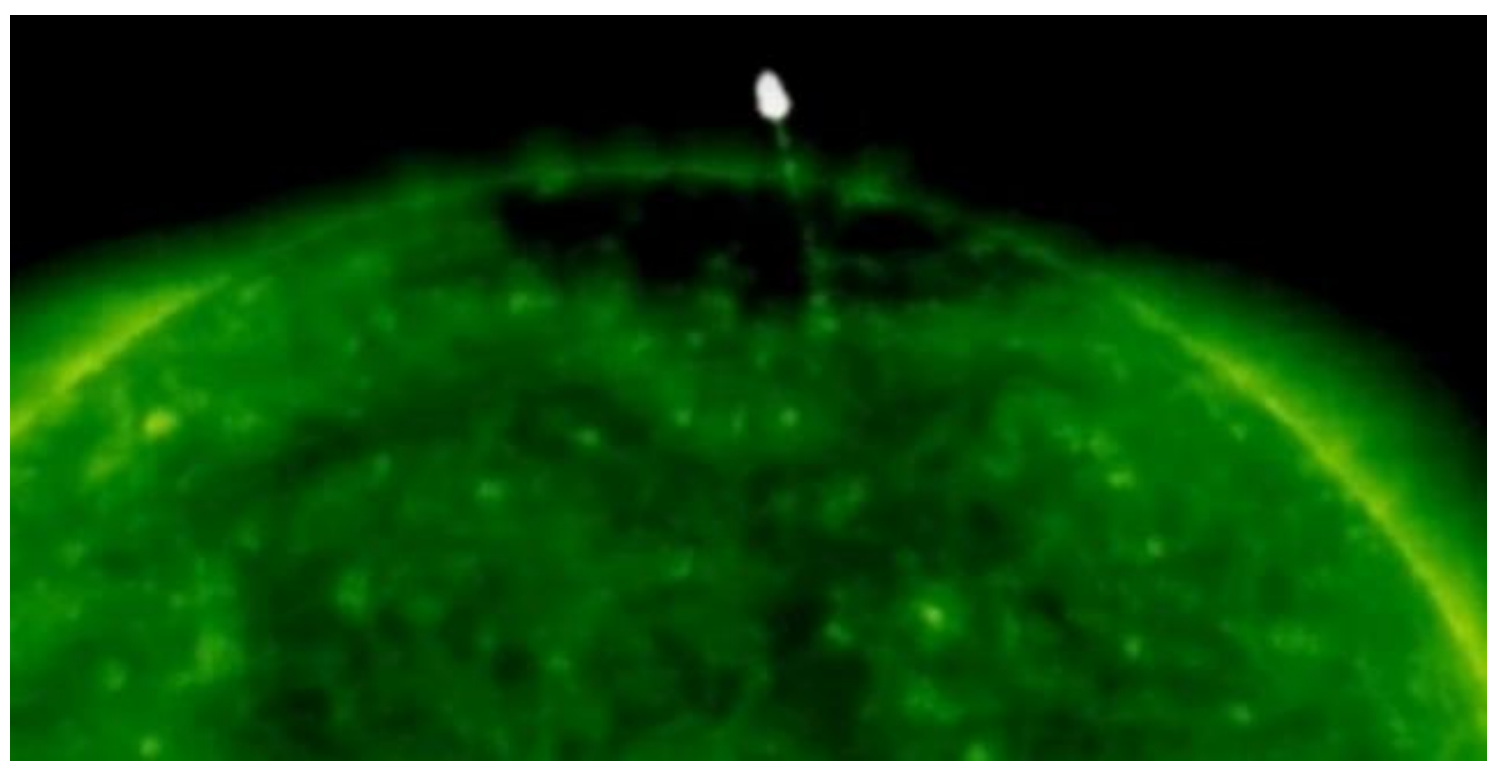

Fig. 4 An earth-sized object flew out of a sunspot region.

by an atmospheric vortex on the proto-sun can make the ambient air temperature exceed $10,000 \times 1000,000^{\circ} \mathrm{C}$, which is higher than 15 million ${ }^{\circ} \mathrm{C}$, and the ambient air pressure be greater than 250 billion atmospheric pressure. Since the atmospheric vortex called sunspot has absorbed sufficient hydrogen, a thermonuclear reaction converting hydrogen into helium can be ignited by such a powerful thunderstorm, which then causes a series of thermonuclear reactions near the sunspot:

$$
{ }_{1}^{2} \mathrm{H}+{ }_{1}^{3} \mathrm{H} \rightarrow{ }_{2}^{4} \mathrm{He}+{ }_{0}^{1} \mathrm{n}+17.6 \mathrm{Mev}
$$

Once a thermonuclear reaction is ignited, a large amount of energy is released in a short time, causing instantaneous heating in local area, generating all kinds of electromagnetic radiation, even appearing a sudden increase of bright spots beside the sunspot, which is the so-called solar flare. Because flares represent the eruption of solar thermonuclear reactions, there will be violent explosions, which may change the structure of sunspots or make them shrink or decay.

Generally, the formation and disappearance of sunspots can only take days to weeks, and a sunspot can only attract a limited range of hydrogen, the hydrogen beyond this scope cannot be processed. So after the recession of a sunspot, without other sunspots to take over, the thermonuclear reactions on the Sun would cease. Fortunately, the Sun has nurtured many planets that can spin around it rapidly, including Mercury, Venus, Earth, Mars, Jupiter, Saturn, Uranus and Neptune, which can help the Sun to produce a series of sunspots that spread all over the Sun, making the Sun produce light and heat continuously. Table 1 shows the ratio of the main planets' gravity on objects on the Sun's surface and the revolution periods of these planets. We can see that Jupiter has the strongest gravitational pull on objects on the Sun's surface, while the revolution period of Venus is approximately nine times the rotation period of the sun.

But among the eight planets in the Solar System, the orbital planes of Mercury, Venus, Earth and Mars do not span the north and south poles of the Sun, only the orbital planes of Jupiter, Saturn, Uranus and Neptune span the north and south poles of the Sun, as is shown in Fig. 5. Since Jupiter is the most massive of the last four planets and the closest to the Sun, it has the greatest effect on the atmospheric vortices called sunspots at the North and South poles of the Sun. In fact, after the first batch of vortices called sunspots are produced at the poles by Sun's rotation, when Jupiter passes through the solar polar sky toward 
Table 1 Ratio of the main planets' gravity on objects on the Sun's surface and the revolution periods of these planets.

\begin{tabular}{lllll}
\hline planet & mass & average distance from the sun & $\begin{array}{l}\text { Ratio of planet's gravity } \\
\text { relative to Mercury's gravity }\end{array}$ & $\begin{array}{l}\text { revolution periods (Solar } \\
\text { rotation period }=25.05 \mathrm{~d})\end{array}$ \\
\hline Mercury & $3.3022 \times 10^{23} \mathrm{~kg}$ & $57909050 \mathrm{~km}$ & 1 & $87.9691 \mathrm{~d}$ \\
Venus & $4.8690 \times 10^{24} \mathrm{~kg}$ & $108209184 \mathrm{~km}$ & 0.42228 & $224.7 \mathrm{~d}$ \\
Earth & $5.9650 \times 10^{24} \mathrm{~kg}$ & $149597888 \mathrm{~km}$ & 2.70684 & $365.24 \mathrm{~d}$ \\
Mars & $6.4219 \times 10^{23} \mathrm{~kg}$ & $227925000 \mathrm{~km}$ & 0.12554 & $686.980 \mathrm{~d}$ \\
Jupiter & $1.9000 \times 10^{27} \mathrm{~kg}$ & $778547050 \mathrm{~km}$ & 31.8327 & $11.8618 \mathrm{yr}$ \\
\hline
\end{tabular}

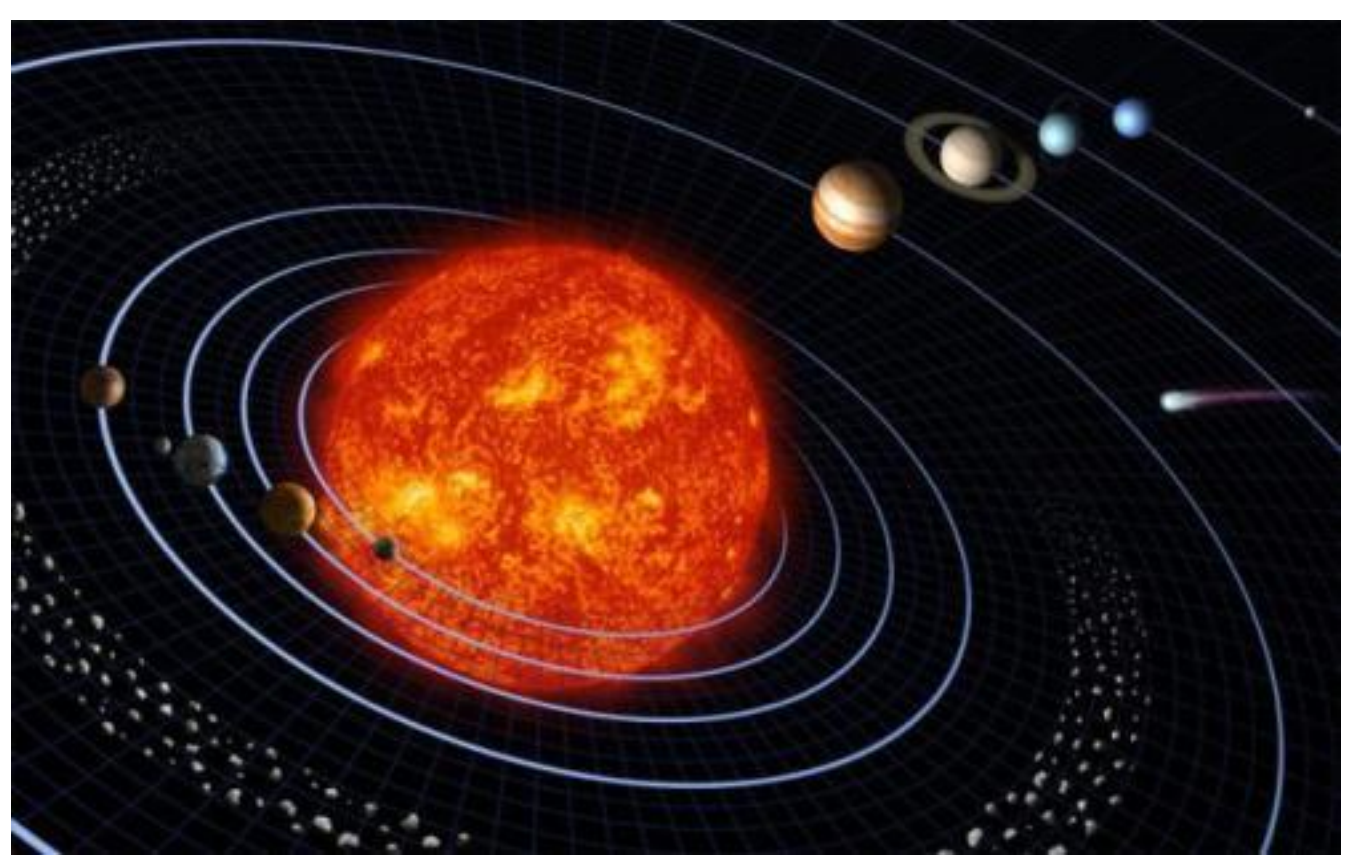

Fig. 5 Orbits of the eight planets in the Solar System.

low latitude, it will attract the inner vortices of the sunspots and bring them to the lower latitudes, just as the moon attracts tide on the Earth. After these vortices absorb enough airflow, they grow into trailing sunspots, continuing the thermonuclear reaction of the leading sunspots. Since Jupiter is very long and big, when Jupiter passes through the solar polar sky toward low latitude, it can not only replicate polar vortices to produce the first generation of trailing sunspots, but also replicate the mature first generation of trailing sunspots to produce the second generation of trailing sunspots, repeat this until near the equator, multiple generations of trailing sunspots can be produced. Then Mercury, Venus and Earth reproduce these sunspots from the north or south poles and currently on both sides of the equator along their orbits, making sunspots surround the world. For example, once Jupiter moved southward over the northern hemisphere of the Sun, it replicated a vortex from the northern hemisphere to a region near the solar equator, producing a sunspot SP1 with its magnetic north pole pointing towards the south pole of the sun and its magnetic south pole pointing towards the north pole of the Sun; another time Jupiter moved northward over the southern hemisphere of the Sun, it replicated a vortex from the southern hemisphere to a region near the solar equator, producing another sunspot SP2 with its magnetic north pole pointing towards the north pole of the Sun and its magnetic south pole pointing towards the south pole of the Sun. If SP2 is to the east of SP1 and adjacent to SP1, then SP1 and SP2 is a couple of sunspots with opposite magnetic polarity. Observed from remote Earth, SP2 is very much like the trailing 
sunspot of SP1. When Mercury, Venus, Earth or Mars passes over the couple of sunspots, it replicates the couple, producing many clusters of paired sunspots, usually distributed along the direction of the planet's revolution. Since Jupiter is the planet that has the greatest impact on sunspots and produces the most sunspots, and Jupiter's revolution period around the sun is 11.8618 years, the cycle of sunspot activity is about 11 years.

When sunspots surround the Sun, the Sun becomes a shining star, which has a surface temperature of $6000^{\circ} \mathrm{C}$ and an internal temperature of 15 million ${ }^{\circ} \mathrm{C}$. It is almost a sphere interwoven by thermal plasma and magnetic fields, but in such a high temperature environment, solar atmospheric vortices can still be generated and active. That is because a sunspot can reach a depth of tens of thousands of kilometers, and a diameter of thousands to tens of thousands of kilometers, which consists of thousands of spiral cloud bands, each spiral cloud band forming a spiral circuit with excellent conductivity and generating a corresponding dipole magnetic field, therefore each sunspot has a strong comprehensive magnetic field with magnetic intensity ranging from 1000 Gauss to 4000 gauss, which is ten thousand times stronger than geomagnetic field. This strong magnetic field can inhibit the transfer of energy from solar interior through convection, therefore, the inner temperature of the sunspot is lower than outside temperature. As air mass swept in by a vortex of the sun sink faster and colder, after a long spiral path, at the bottom of the vortex, the velocity of the airflow is tens of times faster than that of scale 12 typhoon, so the air mass have already condensed into huge crystals. Some crystals are even larger than one Earth, so they are prone to severe friction and collision during rapid rotation, leading frequent and violent thunderstorms, releasing huge amounts of electricity and heat, causing a series of thermonuclear reactions near the sunspot. That is a sunspot on a star.

In fact, not only nuclear fusion but also nuclear fission are taking place in the Sun. Because when light nuclei are fused into heavier nuclei, in addition to the formation of heavy nuclei, superfluous high-energy neutrons are also produced; if there is a certain proportion of heavy nuclei in the reactor of nuclear fusion, the neutrons produced will bombard the heavy nuclei, causing nuclear fission, then generating new light atom; such light atoms can continue to participate in thermonuclear reactions, producing new neutrons, therefore forming chain reactions. For example, in the reactor of hydrogen fusion into helium, after the reaction of tritium with deuterium, except a helium nucleus is formed, there's also a superfluous high-energy neutron; since a sunspot has absorbed a certain proportion of lithium nuclei, the neutrons from nuclear fusion would bombard lithium nuclei, promoting lithium fission to produce a new tritium:

$$
{ }_{3}^{6} \mathrm{Li}+{ }_{0}^{1} n \rightarrow{ }_{1}^{3} \mathrm{H}+{ }_{2}^{4} \mathrm{He}
$$

This tritium can continue to participate in the tritium-deuterium reaction, producing new neutrons, therefore forming chain reactions, providing an endless stream of fuel for nuclear fusion.

\section{The Source of Star Energy}

Because the sun keeps spinning, it can continuously generate polar vortices, forming sunspots at high latitude. Duo to the constant revolution of the eight planets in the solar system, they can replicate sunspots around the Sun. These sunspots not only absorb hydrogen and other interstellar materials in space continuously to supplement fuel for the thermonuclear reactions in the Sun but also sweep great air mass to make them condense into huge crystals, and then make these huge crystals undergo a long spiral path to convert their gravitational potential energy into huge kinetic energy, finally, their kinetic energy is converted into huge electric and thermal energy by crystal collisions, igniting a series of thermonuclear reactions inside and beside the sunspot, releasing immense energy. It can be seen that the Sun's rotation 
energy, gravitational potential energy and the energy generated by nuclear fusion and nuclear fission constitute the source of star energy. Since the mass of the Sun only increases, so does the gravitational potential energy of an object; since the sun spins nonstop, sunspots can constantly absorb hydrogen and other interstellar matter, also, nuclear fission can recover hydrogen, consequently, there is a constant supply of fuel to sustain solar thermonuclear reactions; since the eight planets keep revolving fast, they can reproduce sunspots to continue the thermonuclear reactions of the Sun. Hence, the thermonuclear reaction of the Sun is eternal. But if some fast-moving planets such as Mercury and Venus were swallowed up by the supposed red giant, the thermonuclear reaction on the Sun would stop [10].

\section{Conclusions}

Since the existing hypothesis of solar formation and evolution has serious defects, the author studied again the formation and evolution of the Moon, the Earth and the Sun, and discovered the formation and evolution of the Sun and the real source of star energy. The author could also explain many solar activity phenomena such as sunspots, flares, prominences, etc.

\section{References}

[1] Woolfson, M. 2000. "The Origin and Evolution of the Solar System.” Astronomy \& Geophysics 41 (1): 1-12.

[2] Greaves, Jane S. 2005. "Disks Around Stars and the Growth of Planetary Systems.” Science 307 (5706): 68-71.

[3] Touboul, M., Kleine, T., Bourdon, B., Palme, H., and Wieler, R. "Late Formation and Prolonged Differentiation of the Moon Inferred from W Isotopes in Lunar Metals.” Nature 450 (7173): 1206-9.

[4] Bowring, S., and Housh, T. 1995. “The Earth's Early Evolution.” Science 269 (5230): 1535-40.

[5] Yin, Qingzhu, Jacobsen, S. B., Yamashita, K., Blichert-Toft, J., Télouk, P., and Albarède, F. (2002). “A Short Timescale for Terrestrial Planet Formation from Hf-W Chronometry of Meteorites.” Nature 418 (6901): 949-52.

[6] Lin D. N. C. 2008. "The Genesis of Planets”. Sci. Amer. 298 (5): 50-59.

[7] Tsiganis K. et al. 2005. "Origin of the Orbital Architecture of the Giant Planets of the Solar System”. Nature 435: 459-461.

[8] Pollack J. B. et al. 1996. "Formation of the Giant Planets by Concurrent Accretion of Solids and Gas”. Icarus 124: 62-85.

[9] Li Qibin, and Zhou Hongnan 1997. "The Observation of the Collision of Comet P/Shoemaker-Levy9 with Jupiter in China”. Progress in Astronomy 15 (2): 129-136.

[10] Sack Mann, I. J. et al. 1993. "Our Sun III: Present and Future.” Astrophysics Journal 418: 457-468. 